Horizons philosophiques

\title{
La philosophie et la place publique : Strauss vs Kojève
}

\section{François Raymond}

Volume 8, numéro 2, printemps 1998

Défense et illustration de la vulgarisation philosophique

URI : https://id.erudit.org/iderudit/801075ar

DOI : https://doi.org/10.7202/801075ar

Aller au sommaire du numéro

\section{Éditeur(s)}

Collège Édouard-Montpetit

\section{ISSN}

1181-9227 (imprimé)

1920-2954 (numérique)

Découvrir la revue

Citer cet article

Raymond, F. (1998). La philosophie et la place publique : Strauss vs Kojève.

Horizons philosophiques, 8(2), 63-80. https://doi.org/10.7202/801075ar d'utilisation que vous pouvez consulter en ligne.

https://apropos.erudit.org/fr/usagers/politique-dutilisation/ 


\section{LA PHILOSOPHIE ET LA PLACE PUBLIQUE : STRAUSS VS KOJËVE 1}

En étudiant certains penseurs anciens, je me suis rendu compte que la relation entre la quête de la vérité (la philosophie ou la science) et la société a pu être conçue de la manière suivante : la philosophie ou la science, la plus haute activité de l'homme, c'est la tentative de remplacer l'opinion sur "toutes choses" par la connaissance de "toutes choses"; mais l'opinion est l'élément de la société; la philosophie ou science est donc la tentative de dissoudre l'élément dans lequel la société respire, et ainsi elle met la société en danger. Aussi la philosophie ou science doit rester réservée à une petite minorité, et les philosophes ou savants doivent respecter les opinions sur lesquelles repose la société. Respecter les opinions et accepter celles-ci pour vraies sont deux choses entièrement différentes?2.

II y a quelque chose de labyrinthique chez Leo Strauss. C'est le moins que l'on puisse dire à la lecture de certains des textes qu'il a écrits au sujet de Xénophon. Son idée semble être au premier abord assez simple et elle peut être exprimée directement sous cette forme : il existe une philosophia perennis et celle-ci doit être cachée du regard des indiscrets en raison de son contenu disruptif ou à cause de phénomènes de rejet venant de la censure de telle sorte que ce contenu de sagesse est encrypté dans les textes officiels de cette société. Strauss nous expliquera donc comment les auteurs encodent leurs écrits et comment il y a, en conséquence, deux types de doctrine ou de sagesse, l'une sera exotérique à la fois vulgarisée et vulgarisable et l'autre ésotérique et non vulgarisable.

1. Ce texte a été écrit à l'origine dans le cadre du séminaire de Louis-André Dorion à l'Université de Montréal.

2. Leo Strauss, "Un art d'écrire oublié», Chicago Review, 8.1, 1954, p. 64-75, trad. Nicolas Ruwet, in Poétique, 38, avril 1979 p. 244. 
Évidemment, le contenu ésotérique, le contenu non vulgarisable est celui qui est le plus intéressant car l'autre n'a que la valeur toute provisoire du discours permis par le pouvoir d'une époque donnée et évidemment ce type de discours permis ne concerne pas l'essentiel. Et voilà que nous sommes déjà dans les premiers méandres du labyrinthe. Car c'est en tentant de départager le discours qui se partage et de celui qui ne le fait pas que naît la complication.

Je fais d'abord un retour sur les conditions qui peuvent mener un intellectuel à développer un tel thème, quitte à s'y entortiller inextricablement. Strauss s'éloigne de l'antisémitisme allemand en 1932 et, après un détour en France puis en Angleterre, va s'établir aux États-Unis en 38 . II a donc la nature d'un étranger même si l'on suppose qu'il est devenu citoyen américain. Son enseignement commence à la New School for Social Research de New York et se poursuit à l'Université de Chicago au département des sciences politiques de 1949 à 1968. Après sa retraite il enseignera encore. II meurt en 1973. Sa situation n'est pas sans évoquer celle du poète Simonide donnant des conseils au tyran Hiéron sur la façon de bien mener sa ville et que Strauss discute avec tous les détails dans son commentaire du Hiéron de Xénophon ${ }^{3}$, car Simonide aussi est un étranger reçu avec tous les égards dus à son talent.

Les textes les plus pertinents, et que l'on pourrait dire séminaux, pour notre propos concernant l'usage heuristique que Strauss fait de la notion de contradiction, paraissent à partir de 1939. Ils se présentent sous la forme d'abord d'un commentaire de l'oeuvre La république des Lacédémoniens de Xénophon 4 . Ensuite, Persecution and the Art of Writing paraît en 415. Un autre texte, intitulé On Tyranny, paraît en 1948, c'est un commentaire du Hiéron de Xénophon. Ce commentaire est traduit en français : De la tyrannie paraît chez Gallimard en

3. Leo Strauss, De la tyrannie, Paris : Gallimard, 1954.

4. Cf. L'esprit de Sparte et le goût de Xénophon, ce texte a été traduit par Olivier Sedeyn in Léo Strauss Le discours socratique de Xénophon suivi de Le Socrate de Xénophon, Combas : Édition de l'éclat, 1992, p. 213-242.

5. Social Research, $8.1,1941$. Ce texte sera repris, en 1952, dans un livre du même titre, Glencoe, Illinois : Free Press. 
1954. En plus, cette édition comprend des commentaires d'Alexandre Kojève 6 avec une réponse de Strauss à celui-ci et à d'autres critiques. Également en 1954 paraît On a forgotten kind of writing7. Or, durant la période qui s'étend bien avant 1939 jusqu'à tard dans les années 50, un antisémitisme endémique frappe l'Europe et une partie de l'Amérique comme on le sait. À partir de 1942 un effort de guerre important et, en fait, jamais vu en Amérique, coalise les esprits et exige un consensus autour de certains points de vue politiques liés en contrepartie à des mécanismes de censure. Ce qui aboutira à la guerre froide et à des phénomènes comme le procès des Rosenberg et le maccartisme. On est donc en bonne position pour estimer que durant ces années tout ne pouvait pas être ouvertement discuté, surtout concernant la politique qui, par hasard, est le sujet de prédilection de notre Strauss. Mais, et c'est là la subtilité de la chose, Strauss entreprend de nous montrer comment les choses de la politique peuvent être discutées si elles sont dissimulées dans une recherche sur l'antique politique grecque, en ce temps-là.

Strauss soutient cette thèse avec un point de vue anhistorique. Tous les lecteurs théistes doivent sentir un attrait pour cette rigueur dans le jugement qui tient compte d'un "ordre éternel8». Son écriture doit les stimuler, entre autres, parce que l'on voit poindre une référence à l'absolu. Strauss suppose l'existence d'un absolu de connaissance dont l'épiphanie n'a pas d'histoire. Ce à quoi s'opposera son interlocuteur Kojève qui tient que l'esprit se développe dans l'Histoire, elle-même conçue comme sédimentation du travail humain ${ }^{9}$. Lisant Xénophon sur ce fond éternel, Strauss fera cette interprétation qui vaudra autant pour l'Antiquité que pour l'époque à laquelle il écrit. Ce qui revient à dire que si l'on arrive à énoncer quelque chose de pertinent valant dans l' "ordre éternel" on énonce en même temps quelque chose valant, à plus forte raison, pour un présent donné.

6. Ceux-ci sont la reprise de deux articles parus en 1950 in Critique 41 et 42 : "L'action politique des philosophes (I) et (II)".

7. In Chicago Review, $8.1,1954$, p. 64-75 repris plus tard dans What is politic?, Glencoe : Free Press, 1959.

8. De la tyrannie, Gallimard, p. 329

9. Cf. note 3. 
II est donc compréhensible que Strauss développe le thème de l'écriture entre les lignes comme moyen de communication codé chez des auteurs de l'antiquité et y ait recours lui-même. Car il n'est pas exclu, comme je tenterai de le montrer, que Strauss n'y ait pas recours en marquant ses textes de traces qui cachent "entre les lignes" un contenu encrypté. Ce schème d'écriture et de lecture (qui est en quelque sorte une méthode de lecture) il l'utilise aussi pour lire des auteurs comme Maïmonide ou Spinoza.

Ici on voit que la complication commence dans le fait que, si l'on se fie à ce que Strauss nous affirme de cet "art d'écrire" entre les lignes, un livre entier peut être écrit pour cacher quelques lignes d'une vérité flamboyante. Donc si j'applique cette méthode aux textes de Strauss lui-même, je suis porté à chercher cette vérité et à supposer que ce sont les commentaires de l'ennuyant et pratique Xénophon qui forment l'apparence et le prétexte derrière lequel se cache une vérité éternelle. Car Xénophon, depuis un bon siècle, est délaissé de l'attention des philosophes et des historiens de la philosophie pour un ensemble important de raisons de discrédit qui ont même été compilées par Léon Robin déjà au début du siècle ${ }^{10}$. À cet égard, plus récemment encore, The Oxford Companion to Philosophy de 1995 ne laisse aucune trace de notre ennuyant et terre à terre Xénophon. C'est donc un bon candidat pour celui qui veut discrètement écrire entre les lignes.

Par exemple, quelles peuvent être les raisons de penser que Strauss applique sa propre méthode de lecture à son écriture ou, en d'autres termes, comment dire si Strauss ne nous décrit pas sa propre méthode d'écriture en jetant un regard instruit sur cette apologie que Xénophon fait de la constitution lacédémonienne?

10. Voir Léon Robin, "Les Mémorables de Xénophon et notre connaissance de la philosophie de Socrate", in L'année philosophique, Paris : 1910 et repris plus tard dans La pensée hellénique des origines à Épicure, Paris : PUF, 1942. Dans cet article Robin énumère les neuf raisons pour lesquelles Xénophon est une source incorrecte de la pensée socratique. 
Afin d'examiner cette question, je vais faire référence à certains éléments de la méthode straussienne de débusquement des vérités cachées dans les textes de l'antiquité. II y a d'abord une espèce d'axiome qui fait qu'il rejette le point de vue historiciste et conséquemment, ou en toute apparence de conséquence, il suppose la contemporanéité de tous les textes d'un même auteur. Ceci lui sert à comparer assez systématiquement les différents écrits d'un même auteur à la recherche d'indices qui lui permettraient de débusquer la connaissance encryptée et non vulgarisable. La supposition que tous les textes d'un même auteur sont contemporains fait ainsi apparaître des contradictions entre ce qui a été écrit en un temps et ce qui aura été écrit en un autre temps antérieur ou postérieur'11.

On peut lire, dans ses propres textes, des indices qui montrent des traces de la possible application de cette méthode d'encryptement qu'il trouve chez Xénophon. Le procédé n'est pas tout à fait sûr, cependant, car l'auteur aura toujours pu faire une erreur, c'est-à-dire qu'il ne se sera pas aperçu qu'il se contredisait.

Si les textes n'étaient pas pensés comme simultanés dans la pensée de leur auteur, les indices perdraient beaucoup de leur portée parce qu'avec le temps, justement, on a plus de chance de se contredire à la suite d'un changement. Mais l'importance de la contradiction comme indice, non pas d'une erreur, mais d'un signe de communication secrète, n'est pas discutée clairement par Strauss. Elle joue pourtant un rôle de pointe aussi important dans son exposé sur les lois lacédémoniennes (1939) que dans la présentation d'un antique «art

11. Il est à noter, par exemple, que l'analyse de la fréquence des mots, méthode qu'il utilise aussi et qu'il nomme "emploi de termes caractéristiques" (De la tyrannie, p. 126-129), montre que le mot "comparer" revient souvent, plus précisément 270 fois dans De la tyrannie, dont 187 fois sous la forme "Comparer". Hormis les mots les plus élémentaires que la langue française ne cesse de ramener, très peu de mots de son vocabulaire sont plus fréquemment employés que "comparer" dans les textes qu'il a écrits pour De la Tyrannie. Tout ceci demande à être réinterprété au regard du vocabulaire anglais de Strauss dont je n'ai pas traité. "Comparer" revient surtout dans les notes en bas de page qui commencent par ce mot, d'où la majuscule initiale. 
d'écrire" à laquelle il s'est affairé, à ma connaissance jusqu'en 1959 , donc sur une assez longue période.

La lecture que Strauss fait des textes de Xénophon en débusquant des traces de contradiction pose plusieurs problèmes et, en retour, ces problèmes et les présupposés de cette méthode de lecture, comme je l'ai indiqué, s'appliquent à ses propres écrits.

Strauss a l'art de lire dans les textes de l'antiquité classique, même dans leurs silences. Et cet art a suscité des lectures attentives de personnages d'une stature importante en France tels Alexandre Kojève et Yvon Belaval. Je reviendrai plus loin sur le commentaire que Kojève fait de Strauss. Pour ce qui est de Belaval, il considère que le traitement de la contradiction est assez important chez Strauss pour en constituer une typologie détaillée ${ }^{12}$.

Belaval déclare : «Pour déchiffrer entre les lignes, il faut une lecture minutieuse et une information considérable; et il y faut une méthode qui puisse être démonstrative ${ }^{13}$ ". Belaval suppose deux qualités qu'il semble, à première vue, attribuer à Strauss. Et effectivement à la lecture de ses différents textes on retrouve une minutie et des exposés sur la rhétorique antique ou l'art de la prose dont les détails relèvent de l'érudition. Par exemple, il insiste sur cette idée que le sommet d'une pièce de prose est "ce qui est dit dans le milieu d'un discours". À savoir si ces nuances étaient débattues dans la rhétorique grecque classique du IVe avant Jésus-Christ, mes recherches non extensives n'ont pas trouvé de traces et Strauss ne donne pas ses sources. Je suppose que Belaval lui concède cette érudition, sinon, premièrement, il ne ferait pas cette déclaration que je viens de citer et, deuxièmement, il ne se serait pas donné la peine d'écrire un texte sur un érudit farfelu. Belaval conclura : "Fondamentalement, c'est la contradiction intentionnelle qui est le signe du secret». Et celui-ci d'énumérer les différents

12. Voir "Pour une sociologie de la philosophie», publié en 1952 dans la revue Critique, p. 855-856, où il discute de la méthode straussienne de lecture entre les lignes.

13. Ibid. p. 855. 
critères explicites de la méthode straussienne de débusquement entre les lignes «des pensées persécutées». Voici la typologie qu'a tirée Belaval des textes de Strauss :

Comment peut-elle s'exprimer en se dissimulant?

Plusieurs méthodes sont possibles : 1-espacer les passages contradictoires : $a=b$ au début de l'ouvrage, $a \neq b$ à la fin ; 2- introduire, comme en passant, un ou deux exposés contredisant l'opinion officielle [ ...]; 3- contredire les implications d'une thèse : si $a=b$ et $b=c$, implicitement $a=c$, mais on soutiendra que $a \neq c$ (donc $a \neq b$ ). Maïmonide écrit que l'explication des secrets bibliques est impossible par nature, puis qu'elle est interdite par la loi (elle est donc possible) ; 4- répéter en modifiant comme par inadvertance : supposé $a=b$ et $b=d+e$, répéter seulement que $a=d$ donne à entendre qu'en réalité $a \neq b ; 5$ - insérer entre deux énoncés contradictoires une assertion intermédiaire qui, par elle-même non contradictoire avec le premier énoncé, lui devienne telle par addition ou omission d'une expression en apparence négligeable. $a=b, a \neq d$, $(b=d+e), a \neq b ; 6 \infty$ user de mots ambigus, en sorte que si $a=c$, mais que $c$, tantôt $=b$, tantôt $\neq b$, on ait le choix entre deux conclusions, $a=b$, ou $a \neq b^{14}$.

Mais aussi il faut ajouter la règle par laquelle Belaval résume la pensée de Strauss à ce sujet : «de deux thèses contradictoires, c'est la plus secrète - la moins conforme à l'opinion commune - qui exprime la vraie pensée de l'auteur'15".

Les choses se compliquent car une autre règle de lecture stipule qu' «aucun terme de quelque importance ne doit être employé dans l'interprétation d'un auteur, qui ne puisse être littéralement traduit dans son langage, qu'il n'ait employé ou qui n'ait été d'usage assez commun à cette époque16". Le problème qui surgit s'expose assez aisément. Un savant érudit et minutieux ne peut manquer de remarquer que cette notion de contradiction, très centrale pour le propos de Strauss, a en même temps une histoire. Et dans le cas qui nous occupe, 14. "Pour une sociologie de la philosophie", Critique, p.856.

15. Idem, p. 858.

16. Idem, p. 855. 
c'est-à-dire dans le cas de Xénophon, la proximité de l'origine du problème de la contradiction fait difficulté.

D'abord, dans l'histoire qui précède, il y a Héraclite. Et chez celui-ci la contradiction est affirmée comme la possibilité conceptuelle d'emprise sur l'unité complexe des opposés en perpétuel devenir. La contradiction est loin de faire problème pour Héraclite. Elle est descriptive et compréhensive ou, si l'on veut, c'est un principe d'englobement de la diversité des phénomènes fondamentaux cosmiques. Je vois mal comment la diffusion d'un tel usage héraclitéen de la contradiction chez les Athéniens pourrait faire penser à s'en servir pour encrypter une pensée interdite et secrète.

Les sophistes aussi forment un groupe important de penseurs pré-socratiques qui devaient exercer encore une influence, au moment où Xénophon vit et écrit ou a été formé: grâce à une pensée relativiste ils se font les champions de la contradiction. Selon les sophistes, la contradiction n'est pas problématique puisque c'est justement la puissance de contradiction qui représente ce qui est à enseigner à l'Athénien qui veut réussir. Ce sont ces rhétoriciens qui firent naître les dissoi logoi, les propos qui vont dans deux sens contraires. Ici encore, nous retrouvons la reconnaissance d'une force de contradiction sur l'agora et non sa reconnaissance comme disfonction. Cet usage sophistique est encore plus facilement accessible à Xénophon puisqu'il s'agit de sa propre ville et de son propre temps. Mais se prévaloir de la capacité de défendre un thème et son contraire n'est pas du tout une méthode d'encodage. Évidemment rien de tout ça n'est discuté par Strauss alors que cette question est au cœur même de sa méthode de lecture de Xénophon.

La contradiction commence à apparaître comme une chose à éviter chez Parménide d'abord, puis ensuite chez Platon pour finir par recevoir sa pleine caractérisation, comme un interdit pour cause d'inconsistance, dans la Métaphysique d'Aristote où elle reçoit son substantif qui est "antilogia». Mais l'on sait que ce livre est sans doute posthume et qu'Aristote est lui-même 
postérieur à Xénophon. Ceci exclut donc que ce dernier ait pris sa connaissance aiguë de l'importance d'éviter la contradiction dans celui-là. II reste donc le trio formé de Parménide, Socrate et Platon. Xénophon fait peu de références claires à Platon. Pour ce qui est des deux autres, l'un ne peut pas être soupçonné d'une grande influence sur Xénophon puisque les textes parménidiens ont une apparence intellectuelle, si je puis dire, qui n'est pas tout à fait la même que celle des textes de Xénophon. Celui-ci, en effet, n'a ni cette intransigeance dualiste, abstraite et quasi transcendante que l'on remarque chez Parménide ni sa concision. Pour ce qui est de Socrate la chose est plus compliquée.

Par ce que je viens de dire, Xénophon tiendrait sa connaissance de l'importance indicielle de la contradiction du couple Socrate-Platon. Mais il faut se souvenir que Xénophon fut longtemps éloigné de Socrate et d'Athènes avant d'y revenir pour se défendre du châtiment d'exil qui tombe sur lui, que durant son séjour en Asie Mineure il-n'a pas pratiqué le métier abstrait du philosophe mais celui de mercenaire ou même, avec la retraite des 10000 , de stratège (qui s'en est bien tiré). Ce qui fait que s'il a été exposé à l'importance de la non-contradiction, ce ne le fut qu'avec le temps. C'est-à-dire qu'il a fallu un certain temps pour que naisse chez un individu à l'esprit pratique tel que Xénophon la conscience de la noncontradiction et aussi la reconnaissance de la portée de celle-ci comme principe d'encryptage. Et encore une fois, je le répète, rien de tout cela n'est discuté par Strauss. C'est comme si Xénophon était doué du sens aigu de la contradiction dans un monde où la chose était un acquis encore tout récent. Et ceci ne semble pas complètement vraisemblable.

Bien sûr, le vocabulaire de Xénophon comporte des mots qui font référence au fait de se contredire mais ces mots sont issus d'un contexte de débat et de contestation d'une loi ou d'une action, beaucoup plus que d'une théorie de la noncontradiction stricte. Les expressions qu'utilise Xénophon réfèrent à un débat où des propos plus ou moins compatibles sont tenus en public beaucoup plus qu'à une conscience bien 
définie de l'opposé logique d'une idée comme cela semble être le cas chez Strauss. En somme, c'est comme si Strauss enfreignait lui-même sa règle selon laquelle il ne faut pas user d'anachronisme dans l'interprétation d'un auteur en le pensant dans des termes qui ne sont pas courants à son époque. C'est comme si Strauss confondait des expressions du genre : «je n'ai rien à redire» ou «je n'ai rien à objecter» que nous retrouvons le plus souvent dans les textes de Xénophon et qui ont un sens faible par rapport à la notion de contradiction comme telle et une expression du genre de celle-ci : «je ne peux ou ne veux pas produire la contradictoire». Pour s'en assurer il s'agit d'aller voir dans les Mémorables, où se retrouve pourtant Socrate (qui serait théoriquement une source de conscience de l'importance de la contradiction). Les expressions ont toujours un sens faible et jamais le sens fort17.

De plus, ce qui est pis encore, c'est que ni chez Belaval, qui s'est donné la peine qu'on a vue, ni chez Strauss, qui est le principal intéressé, n'est abordée la question de la possibilité d'une contradiction involontaire. Strauss fonctionne en fait comme si toutes les contradictions étaient strictement volontaires et révélaient toutes un sens consciemment caché. $\mathrm{Ce}$ qui est étrange, si l'on pense à la proximité du «seuil de la contradiction" situé entre le Poème de Parménide et la Métaphysique d'Aristote, qui rendrait cette conscience difficilement intégrée dans l'esprit de Xénophon et qui rendrait vraisemblable l'existence involontaire de la contradiction alors non signifiante. Car on ne peut pas penser que Xénophon était formaliste ou à tendance formaliste. Et la chose, c'est-à-dire se contredire involontairement, est assez courante même chez des penseurs qui ont un respect plus aigu pour la forme. De sorte que, au sens propre, il est à peu près impossible que Xénophon ne se soit pas contredit involontairement puisque la logique n'existe même pas encore. Au moins quelques contradictions seraient involontaires et il importerait de les distinguer de celles qui seraient volontaires et expressives, et ces distinctions Strauss ne les fait pas. La question n'est jamais abordée.

17. Cf. Les mémorables, $1,2,17 ; 2,6,39 ; 3,5,12$ et $4,4,8$. 
Le labyrinthe devient de plus en plus compliqué si nous supposons ses textes comme simultanément disposés dans son esprit et si nous cherchons des contradictions en les comparant. Une contradiction m'est apparue qui concerne une tension entre des déclarations que l'on retrouve dans l'étude de la République des Lacédémoniens et certains contenus du commentaire de l'Hiéron. Ces textes recèlent, comment ne pas s'y attendre, des contradictions qui mènent l'esprit du lecteur à l'idée que leur auteur cache, lui aussi, quelque chose dans cette contradiction et qu'ainsi il écrit quelques secrets entre ses lignes. De plus, une loi logique concerne les matières qui se contredisent : ex falso sequitur quolibet ou ex contradictione sequitur quolibet. Elle date du Moyen Âge et stipule qu'à partir d'une contradiction puisse être déduit n'importe quoi. Ce qui n'arrange pas les choses puisqu'on ne peut pas jouer comme on veut avec une loi logique. À moins que l'on ne soit passé dans l'ordre symbolique qui est fait de polarités contraires, car les symboles sont affublés de valences positives et négatives...

Mais voyons plus en détail comment cette contradiction naît. Strauss se plaît à parler de la noblesse de l'esprit antique qu'il nomme par les qualificatifs composites grecs kalloskagatos (contraction en un mot de "beau et bon" qu'en anglais il traduit par "gentleman" et qu'en français on traduirait par "gentilhomme»). La difficulté consiste à attribuer à cette kallokagatie des qualités qui ne sont pas celles d'un gentilhomme, d'un gentleman, d'où la manifestation d'une contradiction dans le fait de présenter Xénophon comme un gentleman fourbe et ingrat. Dans le cas qui nous occupe, Strauss développe l'idée que Xénophon, dans son texte sur la Constitution des Lacédémoniens, cache une dénonciation en règle de Sparte par la révélation, entre les lignes, de vices importants. De sorte que Xénophon ferait, en apparence, l'apologie de Sparte dont il a reçu un domaine en retour de service rendu, mais qu'en réalité, Strauss, débusquant toutes sortes de contradictions, transforme cette apologie en une dénonciation des graves dépravations dont serait affligée Sparte comme la promiscuité familiale incestueuse et 
l'ivrognerie des femmes. La contradiction réside en ceci, qu'on verra facilement, à savoir qu'un penseur gentilhomme, qu'un gentleman ne peut pas être en même temps ingrat et gentleman. II y a une incompatibilité entre l'ingratitude qui serait l'état de Xénophon par rapport aux Spartiates et l'état de la kallokagatie. Rappelons que les Spartiates, eux, ont été justement reconnaissants des services que Xénophon leur avait rendus en lui donnant un domaine qu'il a géré et habité pendant une vingtaine d'années.

Dans l'interprétation que Strauss fait de l'Hiéron cette règle de la contradiction n'intervient nulle part, c'est comme si Strauss s'était aperçu de cette chose inusitée que je viens de décrire, plus haut, à propos de la contradiction. Cependant il faut dire que, tout comme s'il y tenait encore, il a réédité ses règles de lecture en 1952, en 1954 et en 1959, donc après son texte sur la tyrannie chez Xénophon. Dans celui-ci le mot "contradiction" apparaît mais jamais dans le contexte d'une lecture interprétative d'un sens caché grâce à ce moyen. Et les mots "contredit" ou "contredite" apparaissent toujours dans un contexte où ils sont liés à une négation donc dans le sens d' «objection" plus que dans celui de "contradiction" au sens propre. Par exemple à la page 168 on peut lire :

\begin{abstract}
Si nous considérons, cependant, combien profondément Socrate ou Xénophon sont d'accord avec Simonide au sujet de la tyrannie, nous pourrions être tentés d'attribuer au Simonide de Xénophon la pensée socratique que Xénophon ne contredit nulle part, et suivant laquelle la Sagesse est le bien suprême.
\end{abstract}

La contradiction a encore son importance mais elle ne sert plus à débusquer les vérités cachées mais à établir la cohérence. Alors que si, dans ses textes sur la Constitution lacédémonienne et dans La persécution et l'art d'écrire, Strauss faisait référence à la contradiction c'est qu'elle permettait de mettre le doigt sur une erreur de sens et celle-ci lui semblait cacher-révéler la profondeur de la pensée de Xénophon.

Une erreur de sens d'un autre type se trouve dans De la tyrannie. Cette fois-ci il ne s'agit plus d'une erreur qui se 
trouverait chez Xénophon mais d'une erreur chez Strauss. Car il y a un problème de cohérence dans la définition de la «reconnaissance" qui appartient à la philosophia perennis parce qu'on ne voit pas pourquoi ou comment, d'un point de vue intemporel, l'ingratitude pourrait être l'apanage de l'esprit profond que Strauss attribue à Xénophon.

Je me suis livré à un petit exercice pour voir si Xénophon connaissait la notion de reconnaissance et il est indéniable que cette notion revient très clairement un peu partout dans son œuvre de telle sorte qu'on ne peut pas alléguer que cette qualité n'était pas connue de l'Antiquité grecque et encore moins de Xénophon. D'un autre côté on ne peut pas dire non plus que le défaut de reconnaissance ne fasse pas partie des défauts d'un gentleman selon Strauss parce que celui-ci nous dit clairement que l'ingratitude est une injustice translégale :

Mais, indépendamment de la considération que l'identification du «juste» et du «légal» rendrait impossible la distinction évidemment nécessaire entre lois justes et lois injustes, il y a des éléments de justice, qui, nécessairement, dépassent le cadre de ce qui est légal. L'ingratitude, par exemple, qui n'est pas illégale, n'en est pas moins injuste ${ }^{18}$.

Et à la suite de cette citation, il y a une note qui fait référence à l'Agésilas 4,2 où Xénophon, au sujet du désintéressement du roi de Sparte écrit ceci :

D'ailleurs quand on craint de priver les autres de la reconnaissance qui leur est due, quoiqu'il n'y ait point de recours en justice contre les ingrats, comment se permettrait-on des rapines que la loi défend? Or Agésilas jugeait qu'il y avait de l'injustice non seulement à ne pas payer une dette de reconnaissance, mais encore à ne pas la payer d'autant plus généreusement qu'on est plus puissant.

II est donc assez difficile de penser que Xénophon ne connaissait pas ce sentiment. D'où cette idée que Strauss se contredit clairement à ce sujet et donne à penser qu'il écrit

18. De la tyrannie, p. 145-146. 
entre les lignes lui aussi. Nous sommes pris entre deux contradictions : d'un côté il y a la contradiction entre le fait que, apparemment, Xénophon soit laconisant i.e. pour l'oligarchique Sparte et que, d'un autre côté, il soit, dans les faits, atticisant (selon Strauss) i.e. pour la démocratique Athènes. Mais si nous appliquons à Strauss sa propre règle d'interprétation, nous trouvons une autre contradiction entre une interprétation de la pensée profonde de Xénophon d'une part et d'autre part le fait que Xénophon ne soit qu'un prétexte pour développer certains thèmes sur la politique et la sagesse indépendamment de ces questions de contradiction.

Quant à Kojève, à ce sujet justement, c'est cette dernière voie qu'il emprunte. Pour le voir avec plus de clarté, il faut utiliser la première version du texte intitulé Tyrannie et sagesse qui n'est pas incluse comme telle dans la traduction française de De la tyrannie. Cette première version est parue dans deux numéros 41 et 42 de la revue Critique en 1950. En remaniant ces deux textes pour les inclure dans De la tyrannie, Kojève a supprimé quelques détails de son introduction qu'il est intéressant de restituer. Lui aussi reconnaît l'érudition de Strauss par laquelle celui-ci métamorphose Xénophon : «À travers son interprétation, Xénophon nous apparaît non plus comme l'auteur un peu terne et plat que nous connaissons, mais comme un écrivain brillant et subtil, comme un penseur original et profond" Et Kojève compare la méthode de Strauss à celle du «détective qui, par une interprétation subtile des faits apparents, finit par découvrir le criminel..." Mais il a des réserves. "À vrai dire, la tentation est grande, à la fin, de nier la découverte. En effet, le livre ne peut se terminer, comme les romans policiers, par l'aveu de "criminel" démasqué". C'est pour cette raison que, au cours de son commentaire, Kojève utilise deux fois le nom "Xénophon-Strauss" et plusieurs fois "Xénophon et Strauss". Ensuite, c'est sans autre forme de procès, après avoir déclaré que cette chose est seconde au regard "du problème posé et discuté", qu'il enclenche la discussion des thèmes du Hiéron et des dires de Strauss. Autrement dit, je pense que Kojève aurait discuté le coup même si tout ça n'avait 
été qu'une fiction inventée par Strauss à la manière de certains textes de Borges.

Plusieurs problèmes sont soulevés dans le texte de Kojève, entre autres celui du temps qu'il faut pour faire des modifications aux choses de l'État, celui du motif qui anime le philosophe et le tyran, c'est-à-dire respectivement la reconnaissance et l'amour, celui de l'influence mutuelle du philosophe et du tyran, etc. À cela je pourrais ajouter la question qui ne semble pas débattue très clairement à savoir pourquoi on discute de la tyrannie plutôt que d'un autre mode de gouvernement. Cette question, Kojève y répond d'une certaine façon en mentionnant que la tyrannie est le mode de gouvernement qui serait le plus susceptible d'être pris en charge par le philosophe qui voudrait avoir une influence politique rapide, la raison essentielle pour s'adresser à ce genre d'homme politique étant que le philosophe manque de temps.

De son côté Strauss refuse le dépassement du classicisme, ce qui lui permet de rester à l'aise dans un point de vue intemporel sur les choses politiques. II pense que les classiques ont bien pensé la notion de tyrannie et qu'ils ont eu raison de rejeter la variante moderne de la tyrannie à base d'évolution scientifique et d'homogénéité universalisante des citoyens dans le monde entier qui, selon lui, aurait pour résultat, à terme, de retirer à l'homme la possibilité du dépassement de soi.

Je crois que nous sommes en présence de deux façons très différentes de voir les choses, même si par endroit elles se touchent et concourent. D'un côté Strauss voit les choses de la politique et de la sagesse d'un point de vue qu'il nomme classique. Ce point de vue implique que les choses sont vues sub specie aeternitatis. D'un autre côté, Kojève voit les choses d'un point de vue où la vérité se développe dans l'Histoire. Donc on retrouve ici deux thèses assez opposées. Pour voir cela dans toute sa clarté, je cite Strauss à propos du point de vue classique : "La philosophie au sens strict et classique est la recherche de l'ordre éternel ou de la cause ou des causes éternelles de toutes choses. Je suppose alors qu'il y a un ordre éternel et inchangeable dans lequel l'Histoire prend place, et 
qui n'est, en aucune manière, affecté par l'Histoire ${ }^{19}$ ".

Or cette position on l'a vu être aussi un des traits caractéristiques de cette méthode de lecture qui lui fait voir simultanément les différents textes d'un même auteur. Et l'on voit bien pourquoi. Strauss serait rébarbatif à l'égard de l'historicisme. Et sa position classique et mesurée le mène vers des assertions qui ont un accent tout parménidien. II poursuit cette citation que je viens tout juste de faire ainsi : "Cela laisse supposer, en d'autres termes, que tout "royaume de la liberté" n'est pas plus qu'une province qui dépend du «royaume de la Fatalité»" Cela présuppose, dans les termes de Kojève, que «l'Être est essentiellement immuable en lui-même et éternellement identique à lui-même».

À partir d'un tel point de vue, si tant est que l'on puisse nommer cela un point de vue (notion qui serait sans doute, pour Strauss, trop perspectiviste et même relativiste), il est aisé de comprendre pourquoi il se refuse à considérer, en toute conséquence, l'historicité de la notion de contradiction. Cette notion une fois entrevue, dans son esprit, reste ce qu'elle est dans toute sa pureté éternelle. Cela dit, par contre, on n'explique pas ni ne lève la contradiction qui réside dans l'existence d'un Xénophon ingrat et très averti des usages ésotériques de la contradiction.

Lenjeu de cette confrontation entre ces deux intellectuels du milieu de ce siècle peut être résumé dans deux questions. La première est celle-ci : "un concept peut-il être touché ou affecté par le temps, par son développement dans l'Histoire?" On voit qu'une réponse affirmative à cette question verse du côté d'Héraclite, de Hegel. Cependant, la réponse négative verse du côté de l'éternité du concept, donc dans la direction de Parménide, de Platon et de Strauss.

La deuxième question pourrait être celle-ci : "comment doit-on gouverner les hommes en toute justice? " A cette question Kojève répond par le récit du développement dans l'Histoire humaine de l'idée d'un gouvernement de plus en plus homogène et universel lié au développement des sciences qui 19. Idem. p. 343. 
accorderont graduellement à chacun selon ses dons et ses qualités. C'est une tendance que, grosso modo, je qualifierais de démocratique. Par contre, Strauss pense que le seul gouvernement juste serait aristocratique comme les classiques le pensaient, ce qui sous-entend la thèse selon laquelle une certaine partie seulement des humains aurait accès à la connaissance et l'autre ne pourrait pas y avoir recours. Que la philosophie exotérique est toujours en porte à faux.

Une bonne part de la discussion entre Kojève et Strauss joue sur l'idée de "reconnaissance" qui serait le motif du souverain et du sage ou du philosophe et cette question amène l'idée du nombre d'hommes qui reconnaîtront, c'est-à-dire qu'elle pose le problème de savoir qui, exactement, reconnaît le philosophe dans sa quête de sagesse, qui est le souverain? Kojève remarque qu'il y a une difficulté du moment que le philosophe est seul avec lui-même car s'il reste dans son jardin d'Épicure, seul avec l'évidence de sa sagesse, il n'a pas de moyen de savoir si l'évidence qu'il a trouvée en est bien une, ou si elle n'est pas, au contraire, une pure lubie, une folie dans laquelle il s'est enfermé. De plus, quel serait l'intérêt de s'approcher de la sagesse si cet acquis ne pouvait pas être communiqué aux autres. Pour éviter l'argument de l'enfermement fou, il y a toujours moyen de s'ouvrir à ceux qui sauront apprécier la sagesse du philosophe : jardin d'Épicure où quelques amis viennent de temps en temps ou bien «république des lettres" où des lettrés déjà instruits apprécient la sagesse acquise par notre philosophe. Cependant cette solution aristocratique n'est pas, aux yeux de Kojève, une voie intéressante puisqu'il y a toujours le danger d'une autre forme d'enfermement qui, si elle évite la folie du solipsisme, ne peut éviter le préjugé commun à une chapelle, dont je suppose des exemples connus. Ce préjugé aura peut-être été "vrai» à l'origine mais le temps se sera chargé de le rendre "faux" en modifiant les conditions historiques et sociales. C'est de cette façon que Kojève est amené à penser que, pour éviter ces écueils, l'apprenti sage qu'est le philosophe fera l'épreuve de la place publique et de sa dure historicité. 
En somme, l'enjeu de cette discussion entre Strauss et Kojève consiste à établir la ou les conditions dans lesquelles on peut penser la relation de la politique et de la sagesse. Et, au cours de cette discussion, différents points de théorie politique sont triturés sous les yeux du lecteur. Quant à décider qui, de Strauss ou de Kojève, a raison, je ne sais pas si c'est réellement intéressant. Je ne pense pas qu'un théiste verrait des objections à la pensée de l'Être et d'un ordre éternel. Pour ma part, je pense que, à l'avantage de Kojève, l'univers tout entier est en train de plaider pour l'affirmation d'un devenir héraclitéen du monde. Enfin, la règle logique ex contradictione sequitur quolibet justifie sans doute n'importe quelle interprétation de toutes les contradictions que le lecteur rencontrera chez Strauss, qu'elles soient volontaires ou involontaires. Et, évidemment, cette question des dérivations sans fondement impliquées par cette règle n'est jamais discutée par Strauss.

François Raymond Collège Édouard-Montpetit 